\title{
V. IS Wasit Journal

\section{Influence of Changing Porosity and Height of Fins on Thermal Performance of The Metal Foam Heat Sink: Numerical Investigation}

\author{
Abbas J. Jubear ${ }^{1}$, Aqeel Mtasher Uglah ${ }^{2}$ \\ ${ }^{1}$ Department of Mechanical Engineering College of Engineering-Wasit University \\ Email:abbasaljassani@uowasit.edu.iq \\ ${ }^{2}$ Mechanical Engineering Department College of engineering, Wasit University \\ Email:akeelalzaidy22@gmail.com
}

\begin{abstract}
With the major advances in technology, the extreme need for more powerful and efficient electronic devices and equipment has increased, that is requiring more heat generation as a result. So, it is necessary to find a cooling method commensurate with heat generated. This leads to the use of the foam heat sink which is considered as one of more powerful cooling methods for this purpose. The target of this work is to numerically investigate the impact of changing the porosity and the ratio between height of the fin respect to its length on base temperature and heat transfer coefficient in the case of natural convection conditions. The dimensions of the foam fins were $100 * 10$ in $\mathrm{mm}$ (length * width) and the spacing between fins was $8 \mathrm{~mm}$, while the height of fins was variable with ratio ranging from 0.2 to 2.4 . The porosity of model was changing from 0.95 to 0.71 with fixed pore density of 10 PPI. The investigations have been performed by using ANSYS Fluent software (R16.1) and the model of a local thermal non-equilibrium (LINE) has been used in the analysis where, the temperature of the solid part in foam matrix and the fluid are solved individually in energy equation. The heat fluxes were various from 4 to $30 \mathrm{~W}$, and air has been used as a working fluid. The results showed that the average improvement in base temperature of the heat sink is $16.7 \%$, and the maximum enhancement of heat transfer coefficient reaches about $21.3 \%$, when the porosity reduced from 0.95 to 0.79 . The highest enhancement in base temperature and heat transfer coefficient obtains when the height to length ratio is (2.2) and estimated by $30 \%$ and $83.8 \%$ respectively, compared with the ratio of (0.2).
\end{abstract}

Keywords—Natural convection; porosity; Metal foam; Heat sink; thermal performance

\section{INTRODICTION}

Despite the scientific progress achieved in worldwide, controlling the temperature of electronic components is one of the most difficult challenges facing the electronics industry, especially the chip industry. In order to produce more powerful computers of small size. Therefore, the chips are manufactured with a higher processing power and small dimensions, which means that it has a small surface area to dissipate the heat of the component and therefore the temperature of the chip may rise during work and use [1,2]. It must take in account that the dissipated power of the transistor probably causes that the temperature reaches to the not allowed limit or critical limit and this leads to a serious damage and anther dangers may be one of them is a disability of the system. In spite of, the heat generated in the transistor of computer's and another electronic devices is low, but high density and the large numbers of transistors that installed on a chip cause the accumulation of an excessive heat and thereby occur increasing of temperature. The average failure rate of any electronic component rapidly increases with the increasing in operating temperature for this component. In order to ensure the reliability of the operation and without any damage in the electronic devices, it must to be remove the heat it by cooling systems to prevent thermal failure [3]. There is two conventional methods for cooling the first one based on forced convection and which requires fan to obligate the air on movement, the second one based on natural convection which depend on density difference, in other word the air moves due to buoyancy effect. Both methods have disadvantages because when you need to improve the heat transfer, the increasing is done by increase the air flow across extended surfaces or increase the number of baffles and fins, Therefore any enhancement in heat transfer must be 


\section{Wasit Journal}

accompanied by undesirable defects such as the increasing air speed causes more noise and it needs a powerful fan for this purpose as well as, the workspace, weight of device and pressure losses become large by increase of the fins number[4,5]. One of the most prominent solutions to minimize the occurrence of defects mentioned above is the use of an open-cell foam heat sink. Open cell metal foam can be defined is porous material that it contains multi open paths with irregular structure shape. More than $80 \%$ of the metal foam is consist of an open cell pores. It is usually, the foam made of material that have high thermal conductivity. Metal foam heat sink is much lighter than traditional heat sink, and the contact surfaces area of air flow are higher in the fins made of metal foam. Air flow is fully moved through the pores in addition, the flow is turbulent inside it, even in low speeds, this order leads to higher heat transfer coefficients in the metal foam heat sink [6,7]. Feng et al. [8], carried out an experimental study of natural convection metal foam heat sinks in both orientations. The tested samples made of cupper foam with pore density of 5 pores per inch and porosity of 0.91 . The dimensions of foam fins were $100 \mathrm{~mm}$, length * $10 \mathrm{~mm}$, thickness with different heights $(10 / 20 / 40 / 60 / 80 \mathrm{~mm})$ and the spacing between two adjacent fins was $(2.86 / 5 / 8 / 12.5 / 20 \mathrm{~mm})$. The obtained results showed that the optimum open slot was ranged from $5 \mathrm{~mm}$ to $8 \mathrm{~mm}$, and the best height for fins was $(80 \mathrm{~mm})$, in addition, the horizontal direction gives more enhancement in reducing base temperature better than vertical direction. Hetsroni et al. [9], performed free convection heat transfer experimentally in strips of metal foam with heat generation by using IR technique. Metal foam samples consist of two pore densities 20 and 40 PPI with porosity of $85 \%$ and $90 \%$. The dimensions of tested spacemen were $(22 \times 10)$ in $\mathrm{mm}$ as a cross-section area. The results explained that the heat transfer rate for larger pore sizes 20PPI (fewer pore densities) is greater than the sample with 40 PPI due to higher permeability. Phanikumar, et al. [10] investigated natural convection in metal foams experimentally and numerically. Samples made of aluminum with various pore densities (5-40 PPI) and porosity ranging of $(89 \% \geq \varepsilon \leq 97 \%)$ were used. The dimensions of the metal foam block were: length $6.35 \mathrm{~cm}$ and width $5.08 \mathrm{~cm}$. The maximum of base temperature of plate not excessed $75 \mathrm{C}$. The results showed that the metal foam with 5PPI and porosity of $89.9 \%$ introduced higher heat transfer coefficient amongst the other foam samples. Pradeep, et al. [11], studied heat transfer through aluminum foam metal placed inside a channel in vertical direction experimentally. The pores per inch of foams are 10,20,30 and 45 PPI with various porosities ranged from $90.4 \%$ to $94.8 \%$. The size of samples was: length of $150 \mathrm{~mm}$ with different thickness of 10,20 , and $30 \mathrm{~mm}$. The results explained that when the thickness is increased the heat transfer improved from ( 2 to 4$)$ times compared with empty channel and the maximum drop pressure tack places in sample that it has 45PPI. Zhiguo, et al. [12], investigated heat transfer in heat sink with copper foam metal under natural convection, an experimental study. Different types of samples with various pore density from 10 to 40 PPI and porosity was varied as $(0.90-0.95)$ and the heights of foams were $(10-50 \mathrm{~mm})$ with multi inclination angles of $\left(0,15,30,45,60,75,90^{\circ}\right)$. The indicated that the heat transfer rate increased when the porosity is decreased and reduced pore density, regardless of orientation and heights as well as the sample that it has porosity 0.9 and pore density of 10 PPI with inclination angle range from (60 to 75) gives the higher heat transfer coefficient and the best heat transfer rate. Paknezhad, et al. [13], carried out the influence of foam metal heat sink on a hot surface under natural convection conditions. The dimensions of pieces foam made of aluminum foam were $(10 \mathrm{~mm}$, length $\times 40 \mathrm{~mm}$, width $\times 40 \mathrm{~mm}$, height) with pore density 10 PPI and porosity $92 \%$.The inclination angles of heat sink were $45^{\circ}$ and $90^{\circ}$. The results showed that the best cooling efficiency occurs at inclination angle of $90^{\circ}$ (vertically) and it is estimated about $17 \%$ comparing with $60^{\circ}$.The aim of the study is to improve the thermal performance of the foam heat sink by working on changing the porosity and fin height, thus reducing the base temperature and increasing the heat transfer coefficient of fins.

\section{PROBLEM STATEMENT}

The main problem experienced by electronic components and programmable smart devices lies in the increasing of the temperature during the running and work of those components. Therefore, it is imperative to reduce the temperature of these components to ensure their continued operation and increase their life span by using a heat sink with aluminum foam fins. The current study focused on finding the best ratio between the height of the fin to its length, in addition to the best porosity for foam fins. These two factors are closely related to improving the thermal performance of the heat sink and increasing the efficiency of fins on dissipation. 


\section{V/JIS Wasit Journal}

VOLUME: ( 7 ), NO.:( 3 )

르미므

3. NUMERICAL ANALYSIS

This section contains a discussion of the following sections such as: computational domain, the controlling equations, assumptions, also it includes a mesh generation, the optimum mesh size and boundary conditions. Lastly, the validation of present study with another previous study have been discussed.

\section{- 3.1 Computational Domain}

The various components of the prototype (model) have been created by using ANSYS R16.1 package. Where, this component involves six of aluminum foam fins, base plate, and a box. These components are created individually and therefore assembled. The fins configuration and base plate were put within the middle of the domain and after then the bounding box (domain) was constructed as shown in figure 1. The foam fins were put on base plate made of aluminum with size $(100 * 100 * 3.5) \mathrm{mm}^{3}$. The boundary conditions of the process regarding to enclosure domain were placed far from the fins to prevent any flow-reversal throughout the simulation sufficiently. A previous study was adopted for the selection of the fins foam dimensions as mentioned in [8] .The geometrical dimensions of the models and some of the properties of the metal foam fins and air are listed in Table 1.

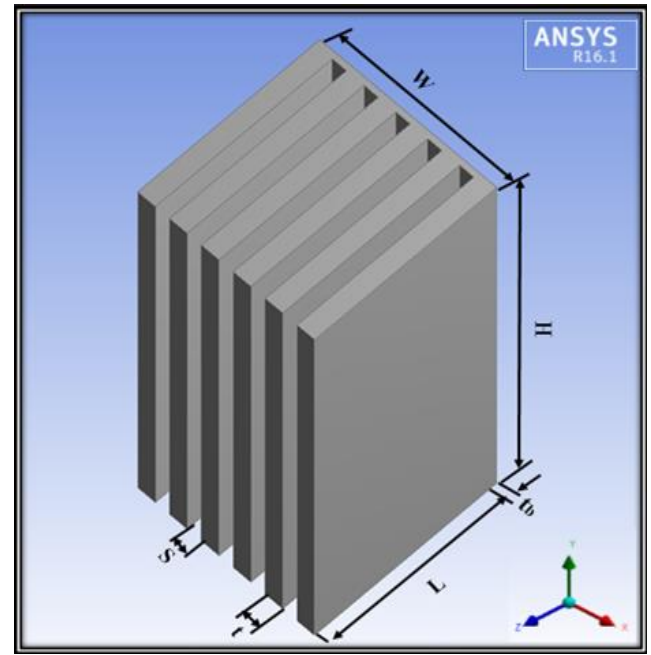

a

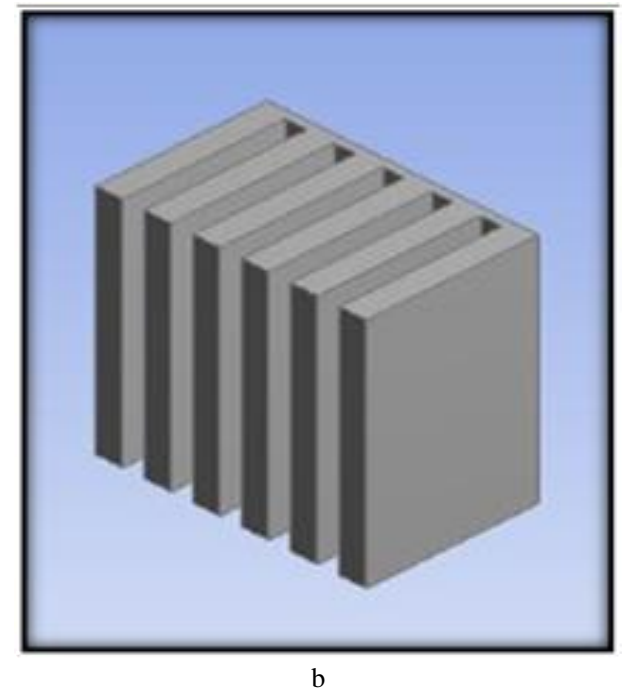




\section{N.T Wasit Journal}

VOLUME: ( 7 ), NO.: ( 3 ) 르미모

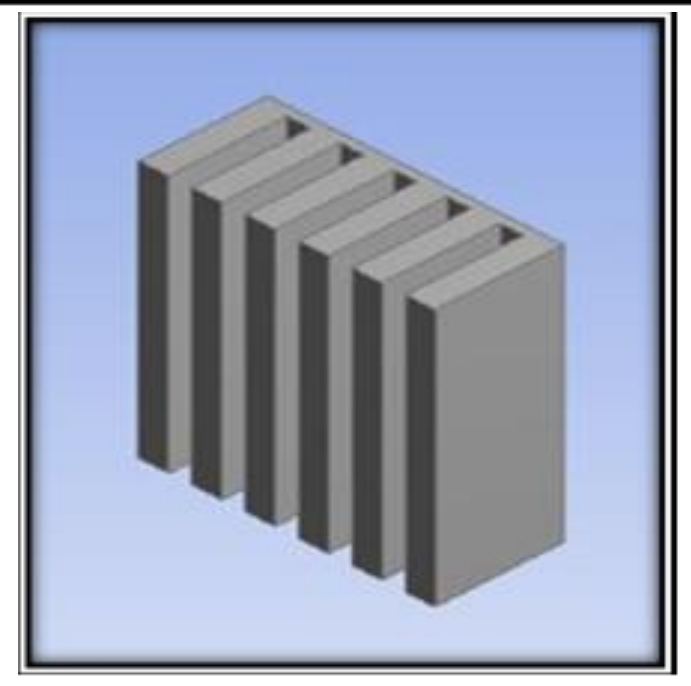

$\mathrm{c}$

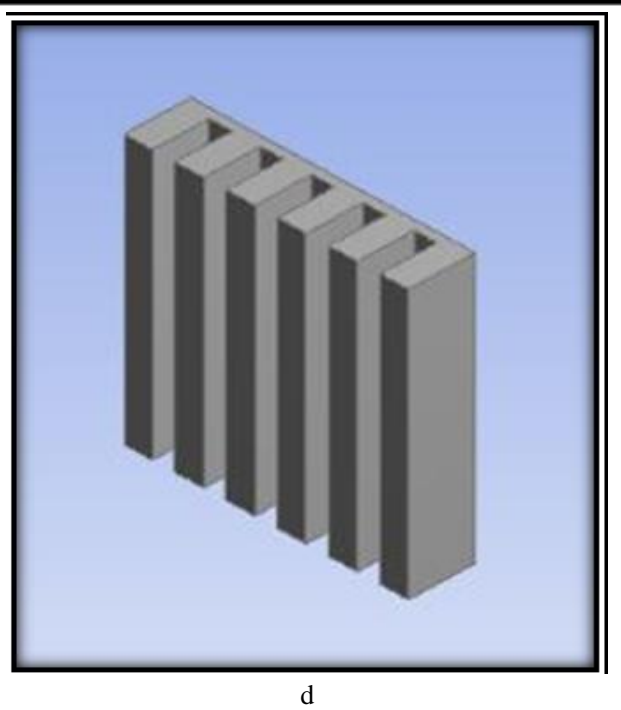

Fig.1. Aluminum Foam Fins with $(H / L) ;(a)=1.0,(b)=0.6,(c)=0.4,(d)=0.2$.

- $\quad$ TABle 1. Geometrical Parameters Properties of Metal FoAm AND Air

\begin{tabular}{|c|c|c|c|c|c|}
\hline \multirow[b]{2}{*}{ Aluminum foam } & PPI & Porosity $(\varepsilon)$ & Ks (W/m.K) & Length (L) mm & 100 \\
\hline & $5,10,20$ & $95 \geq \varepsilon \geq 71$ & 180 & $\begin{array}{c}\text { Height/Length } \\
\text { H/L }\end{array}$ & 0.2 to 2.4 \\
\hline \multirow{3}{*}{ Air } & $\mathrm{K}_{f}$ & $\operatorname{Pr}$ & $T_{a m b}$ & Thickness(t) mm & 10 \\
\hline & \multirow[t]{2}{*}{0.0265} & \multirow[t]{2}{*}{0.7} & \multirow[t]{2}{*}{$21.5^{\circ} \mathrm{C}$} & $\begin{array}{c}\text { Fin spacing (s) } \\
\text { mm }\end{array}$ & 8 \\
\hline & & & & Number of fins & 6 \\
\hline
\end{tabular}

\section{○ 3.2 Assumptions}

For solving the governing equations of the flow and heat transfer such as continuity, momentum and energy equations and for the purpose of limiting the problem, the researcher must put sets of assumptions.

- The type of the flow is laminar.

- The simulations perform under steady state conditions.

- Boussinesq approximations that concerning with change the density and temperature were used.

- With respect to fluid is to be incompressible.

- Ignore internal heat generation and neglect the radiation.

- The model of a local thermal non-equilibrium (LINE) has been used in the analysis where, the temperature of the solid part in foam matrix and the fluid that passing through it are solved separately in energy equation.

\section{- 3.3 Governing Equations}

The governing equations that are used in the ANSYS software, it consists of the continuity, momentum as well as the energy equations. 
3.3.1 Conservation of Mass

It defines as the flowing mass entering into a specific control volume it must be equal to the mass outside from it, and this principle can be indicated by this relation:

$$
\frac{\delta \mathrm{Vx}}{\delta x}+\frac{\delta V y}{\delta y}+\frac{\delta V z}{\delta z}=0
$$

Where: $\mathrm{V}_{\mathrm{x}}, \mathrm{V}_{\mathrm{y}}$, and $\mathrm{V}_{\mathrm{z}}$ are velocity component in the direction $\mathrm{x}, \mathrm{y}$, and $\mathrm{z}$ respectively.

\subsubsection{Momentum Equations}

The other controlling equations that its governor fluid flow is derived from second law of Newton, it named the NavierStokes equations or the conservation of momentum equations. for incompressible fluids the full following equations are taken in this formula: when the effect of gravity is exists in $-\mathrm{y}$ direction, the body force vector become $\mathrm{g}=[0,-\mathrm{g}, 0]$. The momentum equations:

$$
\frac{\rho}{\varepsilon^{2}}\left(V_{x} \frac{\delta V_{x}}{\delta x}+V_{y} \frac{\delta V_{x}}{\delta y}+V_{z} \frac{\delta V_{x}}{\delta z}\right)=-\frac{\delta p}{\delta x}+\mu_{e}\left(\frac{\delta^{2} V_{x}}{\delta x^{2}}+\frac{\delta^{2} V_{x}}{\delta y^{2}}+\frac{\delta^{2} V_{x}}{\delta z^{2}}\right)-\frac{\mu}{K} V_{x}|\vec{V}|-\frac{\rho F \varepsilon}{\sqrt{K}} V_{x}
$$

In X-direction

$$
\frac{\rho}{\varepsilon^{2}}\left(V_{x} \frac{\delta V_{y}}{\delta x}+V_{y} \frac{\delta V_{y}}{\delta y}+V_{z} \frac{\delta V_{y}}{\delta z}=-\frac{\delta p}{\delta y}+\mu_{e}\left(\frac{\delta^{2} V_{y}}{\delta x^{2}}+\frac{\delta^{2} V_{y}}{\delta y^{2}}+\frac{\delta^{2} V_{y}}{\delta z^{2}}\right)-\frac{\mu}{K} V_{y}-\frac{\rho F \varepsilon}{\sqrt{K}} V_{y}|\vec{V}|+\rho g \beta\left(T-T_{\infty}\right)\right.
$$

In Y-direction

$$
\frac{\rho}{\varepsilon^{2}}\left(V_{x} \frac{\delta V_{z}}{\delta x}+V_{y} \frac{\delta V_{z}}{\delta y}+V_{z} \frac{\delta V_{z}}{\delta z}\right)=-\frac{\delta p}{\delta x}+\mu_{e}\left(\frac{\delta^{2} V_{z}}{\delta x^{2}}+\frac{\delta^{2} V_{z}}{\delta y^{2}}+\frac{\delta^{2} V_{z}}{\delta z^{2}}\right)-\frac{\mu}{K} V_{z}|\vec{V}|-\frac{\rho F \varepsilon}{\sqrt{K}} V_{z}
$$

In Z-direction

\subsubsection{Energy Equation}

Energy equation: it divided in two parts, the first part for the fluid in the porous medium (LTNE).

$$
\begin{gathered}
\rho_{f * C_{p_{f}}}\langle v\rangle \nabla T_{f}=\nabla\left\{\left(k_{f e}+k_{d}\right) \cdot \nabla\left\langle T_{f}\right\rangle\right\}+h_{s f} a_{s f}\left(T_{s}-T_{f}\right) \\
\quad \text { Energy equation for the solid matrix (LTNE): } \\
0=\nabla \cdot\left\{k_{s e} \cdot \nabla\left\langle T_{s}\right\rangle\right\}-h_{s f} a_{s f}\left(T_{s}-T_{f}\right)
\end{gathered}
$$

\subsection{Mesh Generation}

FLUENT is define as a finite volume numerical software. When the solution is performed numerically that's it requires meshing for the geometry as shown in figure 2. There is an important issue that the accuracy of the solution is depend on it, which is represented by the sizes of the cells. Where the relation between the accurate of the solution and size of meshes is inverse. because of the smaller mesh size introduces more accurate for solution. But whenever the sizes of the meshes decrease the number of meshes increase too. if the number of sizes become large this lead to require wide computing capacity and the solution take more time. Therefore, it is imperative for the researcher to find a suitable solution to this obstacle. more and more reduction of the cells size has a disadvantage because it requiring a large 


\section{VNJIS Wasit Journal}

VOLUME: ( 7 ), NO.: ( 3 ) 르미므

computer memory. In FLUENT package there are two kinds of grids consist of the tetrahedron and hexahedron cells or a combination between them in 3-D. The choice of the mesh depends mainly on the application type. with respect to applications that have complex geometry, the grids of it consist from hexahedron cells, it can be there is a loss of time for reaching to the correct solution. thereby, the time of the setup is considered the major reason for using unstructuredgrids that it involves tetrahedron cells.

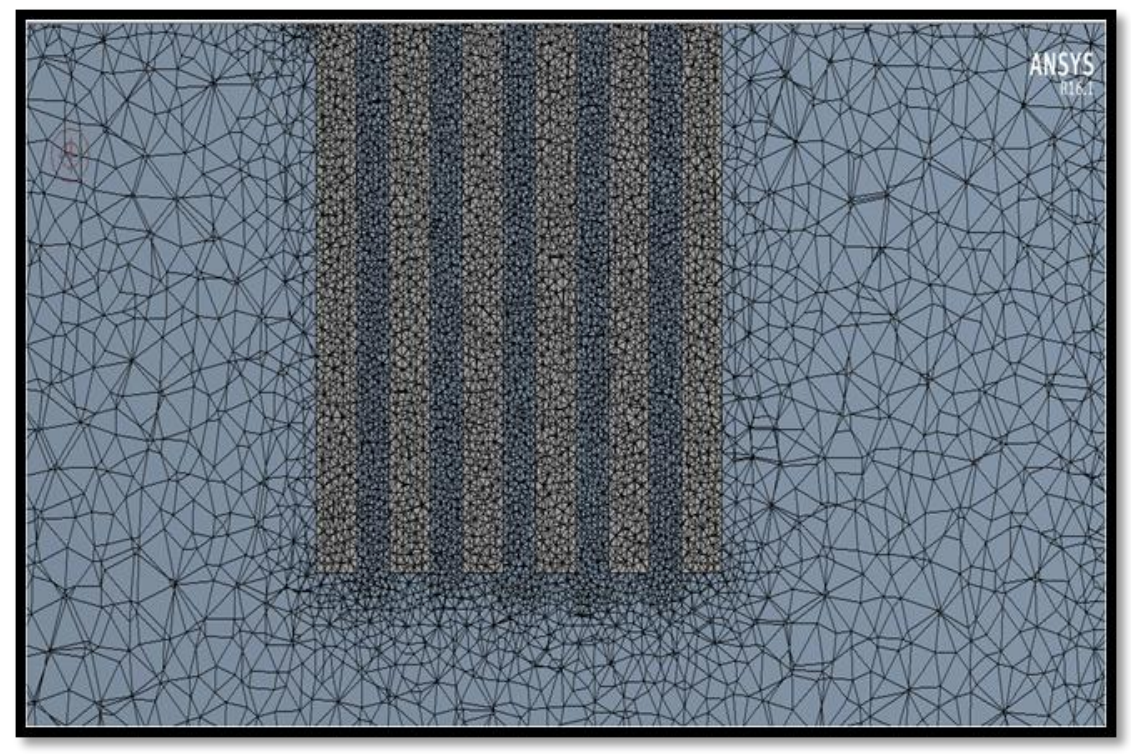

a) Fig. 2. Computational Grid For Metal Foam Fins.

\subsection{The OPTIMUM MESH SIZE PROCEDURE}

For investigating in the spatial mesh resolution and stability on the best mesh which in turn achieves the best results. Where five types of different meshes were created using ANSYS fluent (R16.1) software, see Table (2). The independence of the mesh was investigated through the study of five various mesh sizes with different values of heat input.

- TABLE 2. OVERVIEW OF MESH MODELS

\begin{tabular}{|c|c|c|c|c|c|}
\hline Mesh & M1 & M2 & M3 & M4 & M5 \\
\hline Total Mesh Cells & 222,727 & 260,777 & 785,972 & $2,122,579$ & 3,598439 \\
\hline
\end{tabular}

The results of the study of grid independence of the fins are shown in figure 3. After completing the simulation of the five types of network sizes, the temperature values are then monitored. The fourth and fifth meshes achieved a great similarity in temperature for same heat input, unlike the other three types. Therefore, the fourth mesh was used in the 
simulation to get to know the characteristics of the flow within the domain as well as to capture these characteristics within the fins also.

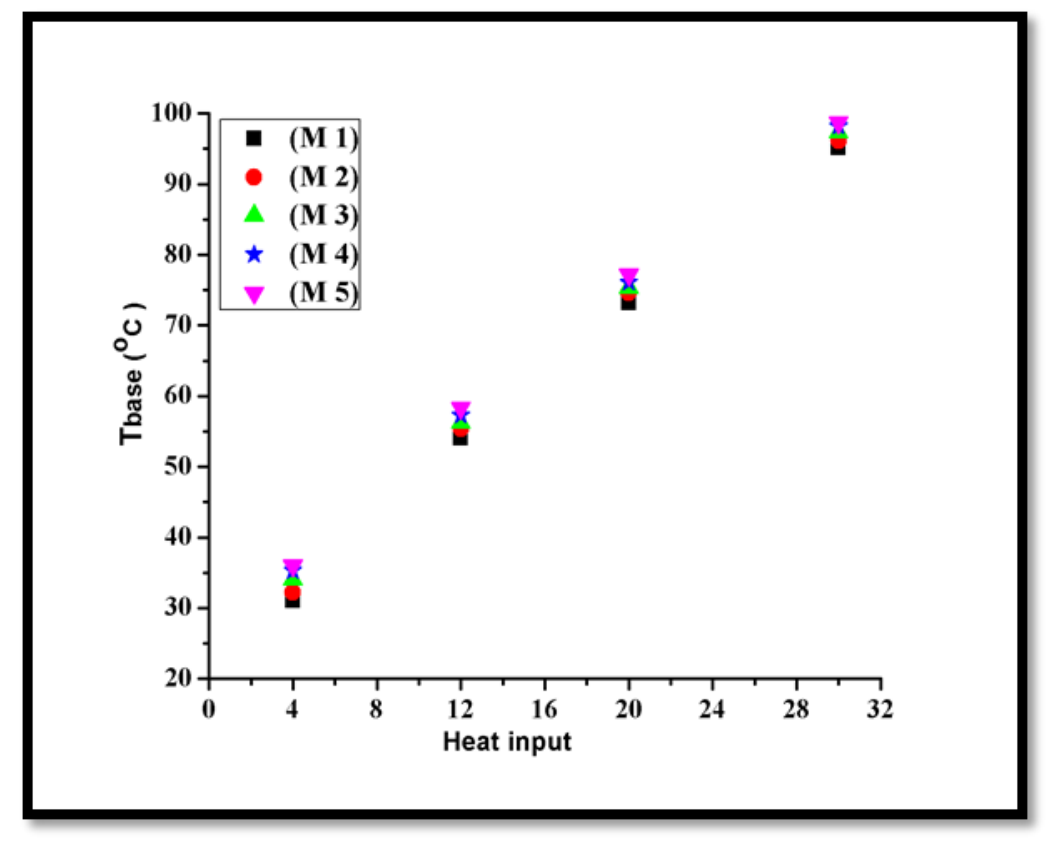

b) Fig.3. Mesh Independent of Numerical Model.

\subsection{Boundary Conditions}

In this study the type of the flow is laminar. The density effect variation with temperature was estimated through the simulation by using the Boussinesq approximation. There are various values of heat input $(4,8,12,16,20,24$ and $30 \mathrm{~W})$ were used. for capturing the behavior of the flow with high accuracy, a very soft border layers mesh sizes were used for zones close to the fins surface. The main condition of the fins model in the base of the fins is a constant heat flux. As well as the boundary condition of the model has been applied at the entrance of the domain (enclosure) is the inlet velocity, where the velocity at the bottom of the fins array is zero. the other three sides of the enclosure are applied at the pressure outlet in other words the fins become under the effect of the atmospheric pressure. The heat transmitted across the fins base by conduction, while at the surface of the fins, the heat is transferred by convection as shown in figure 4 . 


\section{N. IS Wasit Journal}

VOLUME: ( 7 ), NO.: ( 3 ) 르미모
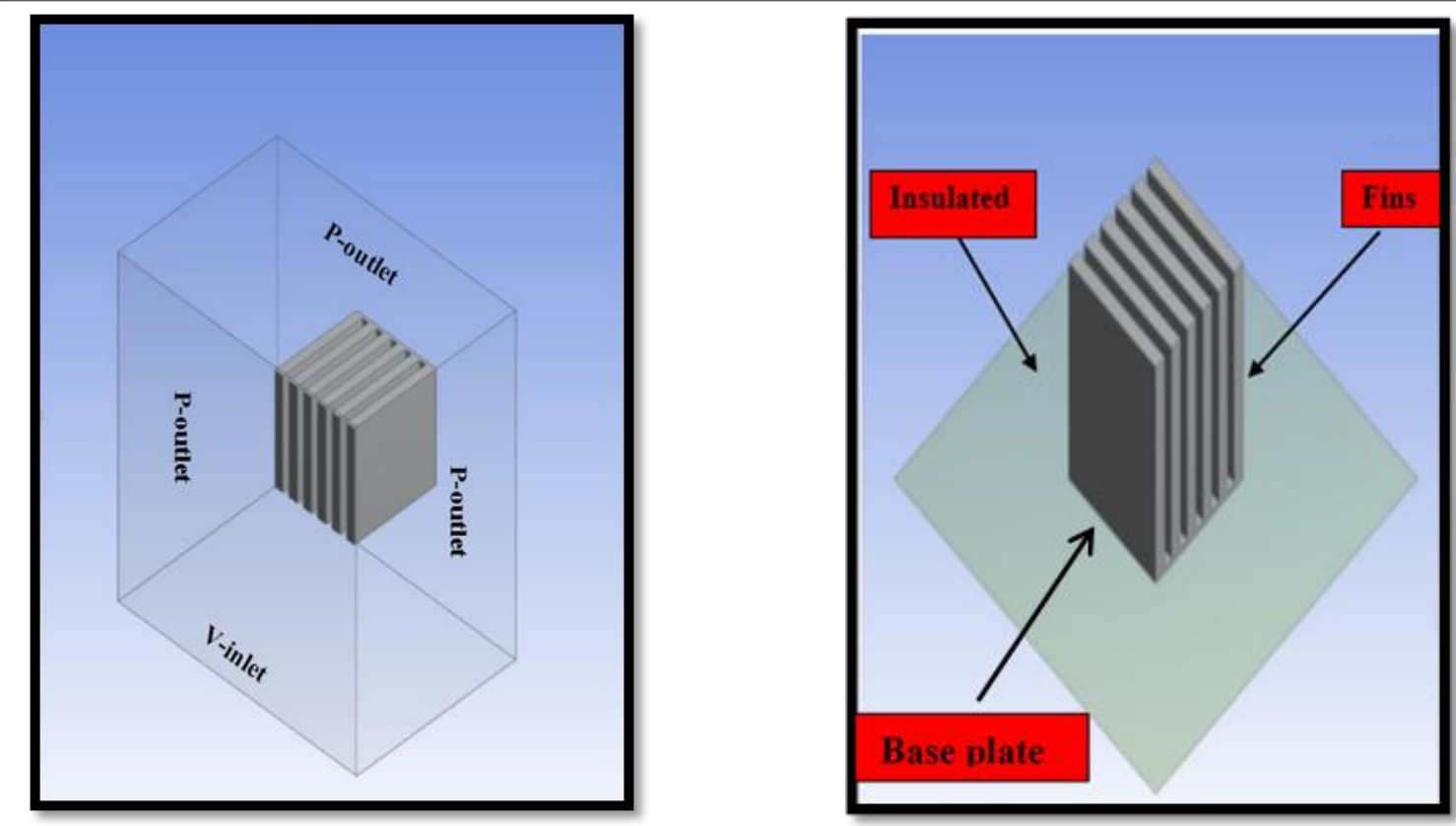

c) Fig.4. Boundary Condition For Domain.

\subsection{Convergence}

The residuals can be defined as the difference between two successive values (iterations) in an equation. The convergence is considered a criteria that it used to finish the solution when the solution reaches the values that are specified by the user, where the residual of iterations for the equations are compared with a values that it determined by user, if the residuals are less than the values which the user-specified the solution deemed as converged and the program stops the analysis as shown in figure 5. 


\section{N.J Wasit Journal}

VOLUME: ( 7 ), NO.: ( 3 ) 르미므

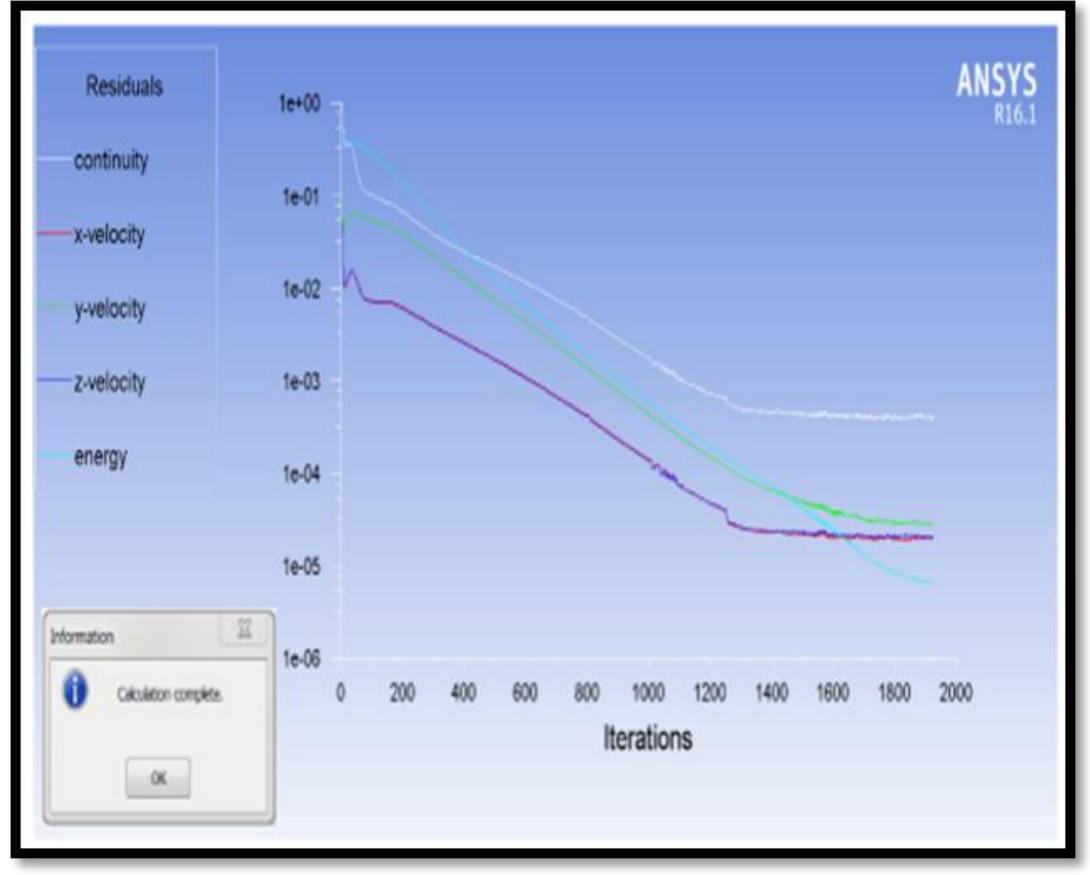

Fig.5. Convergence History to Solve Discrete Conservation Equations.

\section{RESULTS AND DISSUCATION}

This suction includes the validations of present work, in order to compare what was reached during the current study with another study published. As well as discuss the temperatures distribution during the foam fins of the heat sink and display in a form temperature contours.

\subsection{Validation Of The Present Work}

The results of numerical simulation of current study have been validated with experimental results of Feng et al., 2017 [8] as shown in the figure 6. From the figure, there appears to be an excellent agreement between the numerical results of this study and the experimental results of Feng, where the error does not exceed $5.1 \%$. This validation has been performed with copper foam fins that have pore density of 5 and porosity of $91 \%$, as well the size of fins was; length $100 \mathrm{~mm}$, width $10 \mathrm{~mm}$, 


\section{V/JIS Wasit Journal}

VOLUME: ( 7 ), NO.: ( 3 ) 르미므

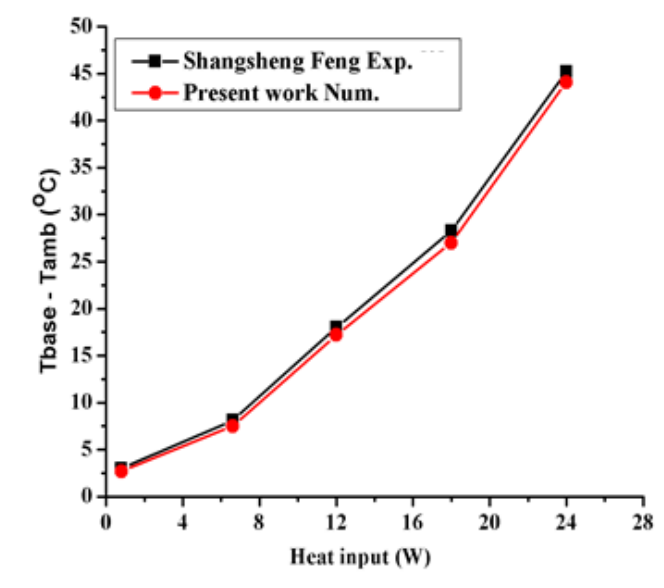

Fig. 6. Validation of Numerical Analysis Graph.

\subsection{The Influence Of Porosity On Base Temperature}

The average base temperature to ambient is plotted with multi values of heat input varied from (4 to 30$) \mathrm{W}$ as shown in figure 7.The comparison was based on the stabilization of the pore density, which was equal to 10 PPI with different values of porosity that ranged as $(0.95,0.91,0.87,0.83,0.79,0.75$ and 0.71$)$.Porosity is one of the most important factors that directly affect on the transferring of heat during the internal structure of metal foams. It is can be notice in curves, when the porosity is small the average base temperature to ambient become small also until the porosity value of 79 , and this behavior is reversed when porosity is 71 , this meaning the highest average temperature tack places at the porosity of $71 \%$ because the effective thermal conductivity of metal foam starts decreasing at this value , and the lowest average temperature occurs at porosity of $79 \%$. This can be explained that the reduced in porosity value increases the effective thermal conductivity of foam metal. Where the average improvement rate in base temperature when reduced porosity from $95 \%$ to $79 \%$ is about $16.7 \%$.

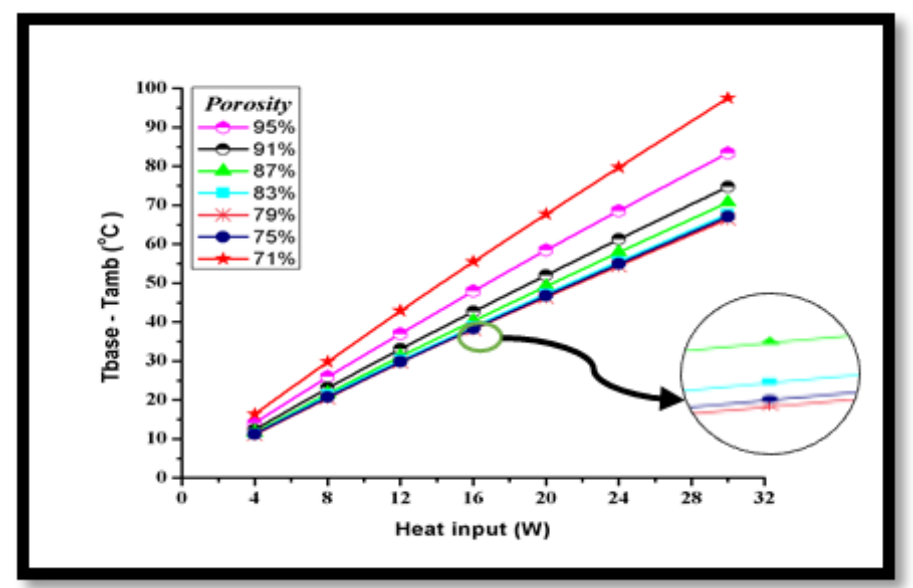

Fig. 7. Variation of average base temperature to ambient with heat input of different porosities 


\subsection{The Influence of Porosity on The Heat Transfer Coefficient}

The heat transfer coefficients are plotted as a function of various heat input with different porosities ranging from $95 \%$ to $71 \%$ as shown in figure 8 . As expected, the heat transfer coefficients increase when there is decreasing in porosity of open-cell foam at certain values of porosity. It can be understand that the higher heat transfer coefficients are obtained when the porosity value is $79 \%$ due to the much reduction in base temperature to ambient occurs at same porosity, and conversely, the lowest values for these coefficients at porosity $71 \%$ because the inversion point in the process of improving thermal performance is with this value $71 \%$,due to the effective thermal conductivity is at the worst value at the stated porosity. The indication of this behavior can be explained. Firstly, because the decreasing in porosity is generate an increasing in the effective thermal conductivity of metal foam .Secondly, the thickness of solid ligaments fibers is increase when porosity is reduced to a certain extent and leading to a significant enhancement in heat transfer by conduction through the fibers and this contribute greatly to improving heat transfer coefficients.

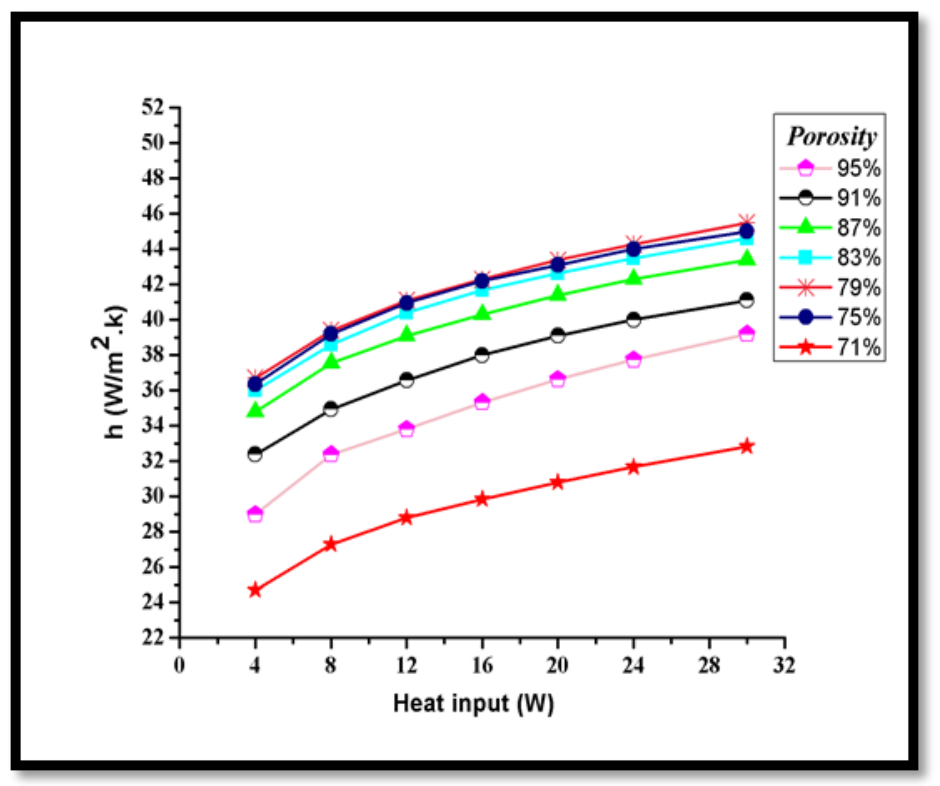

Fig. 8. Variation of Heat Transfer Coefficient as a Function of Heat Input of Various Porosities

\subsection{Influence the Fin Height on Base Temperature}

The figure 9 explains the variations between average base temperature to ambient and heat input for various sets of fin length relative to its heights ranging from 0.2 to 2.4. The first thing that can be observed in figure that the temperature difference increases when the values of power input increase also, quite the contrary, the average of base temperature to ambient reduces when the fin height augments while all other parameters stay constant. This demeanor attributed to the biggest heat exchange area between fins and air surrounding's obtained at ratio of 2.2 where the enhancement of temperature difference estimate by $30 \%$ greater than the ratio of 0.2 . After that when the ratio reaches (2.4) the 


\section{V/J If Wasit Journal

improvement remains constant. Based on the results above, (2.2) was chosen as the optimum ratio for foam fins which gives the best thermal performance

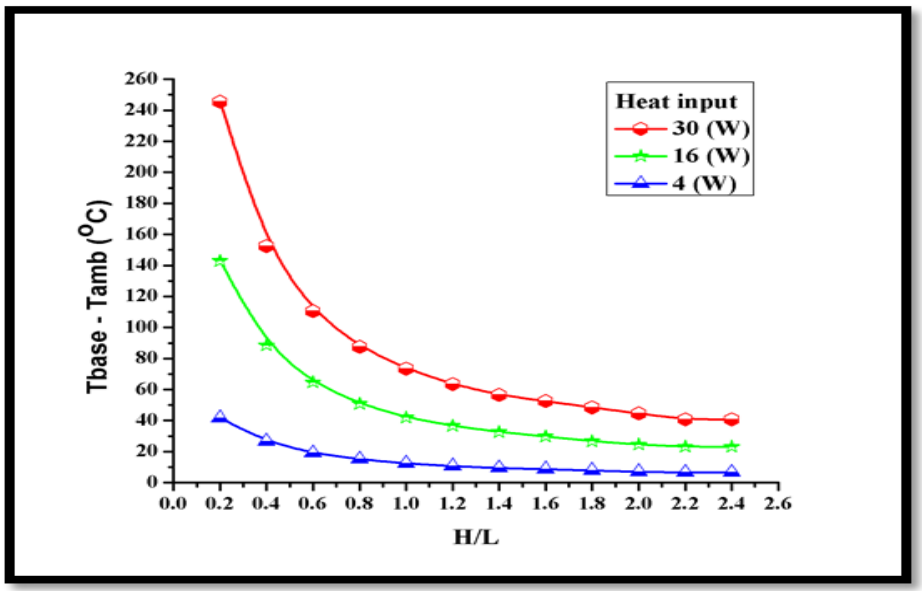

Fig. 9. Variation of Average Base Temperature to Ambient with Heat Input for Multi Values of Fin Height Relative to Length

\subsection{Influence the Fin Height on Heat Transfer Coefficient}

The heat transfer coefficient is draw as a function of different values heat input for various ratios of length of the fin relative to its heights that it ranged from $(0.2$ to 2.4$)$ as shown in figure 10. As known the heat transfer coefficients of the fin depends mainly on the fin height as well as, the important parameter which has prominent effect on heat transfer coefficient is the difference between base temperature and ambient temperature, so the increase in this ratio is contribute significantly for increasing the heat transfer coefficients as it be can explained in the figure .When the ratio is $(0.2)$ the heat transfer coefficients are small compared to the ratio (2.2), after than the enhancement of heat transfer coefficient remains constant at the ratio reaches (2.4). The main reason behind this behavior is the larger heat transfer area for exchange between fins and air around it's, within the ratio is 2.2. According to these results, the best ratio between the length of the metal foam and its heights is (2.2).

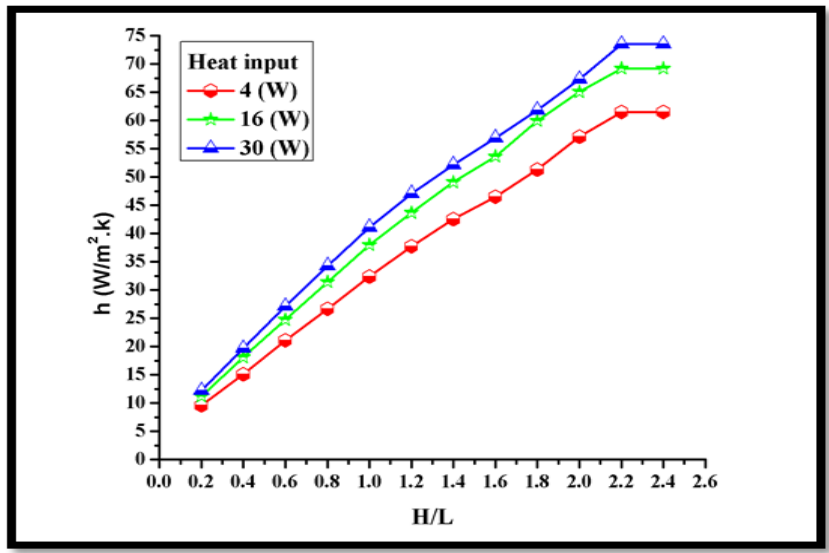

Fig. 10: Variation of Heat Transfer Coefficient as a Function of Various Fin Height Relative to Length.

\subsection{TEMPRATURE CONTOURS}




\section{VNJIS Wasit Journal}

VOLUME: ( 7 ), NO.: ( 3 ) 르미미

In order to demonstrate the effect of the changing in porosity value on the base plate temperature for metal foam heat sink. A numerically comparison between two samples that have same PPI with constant heat input has been conducted. It is noticed when the porosity is reduced that's the base temperature of heat sink is also reduced. Figure 11 gives a complete visualization of heat flow through the surfaces of the fins and introduces an excellent indicator in improving the thermal performance of the heat sink.

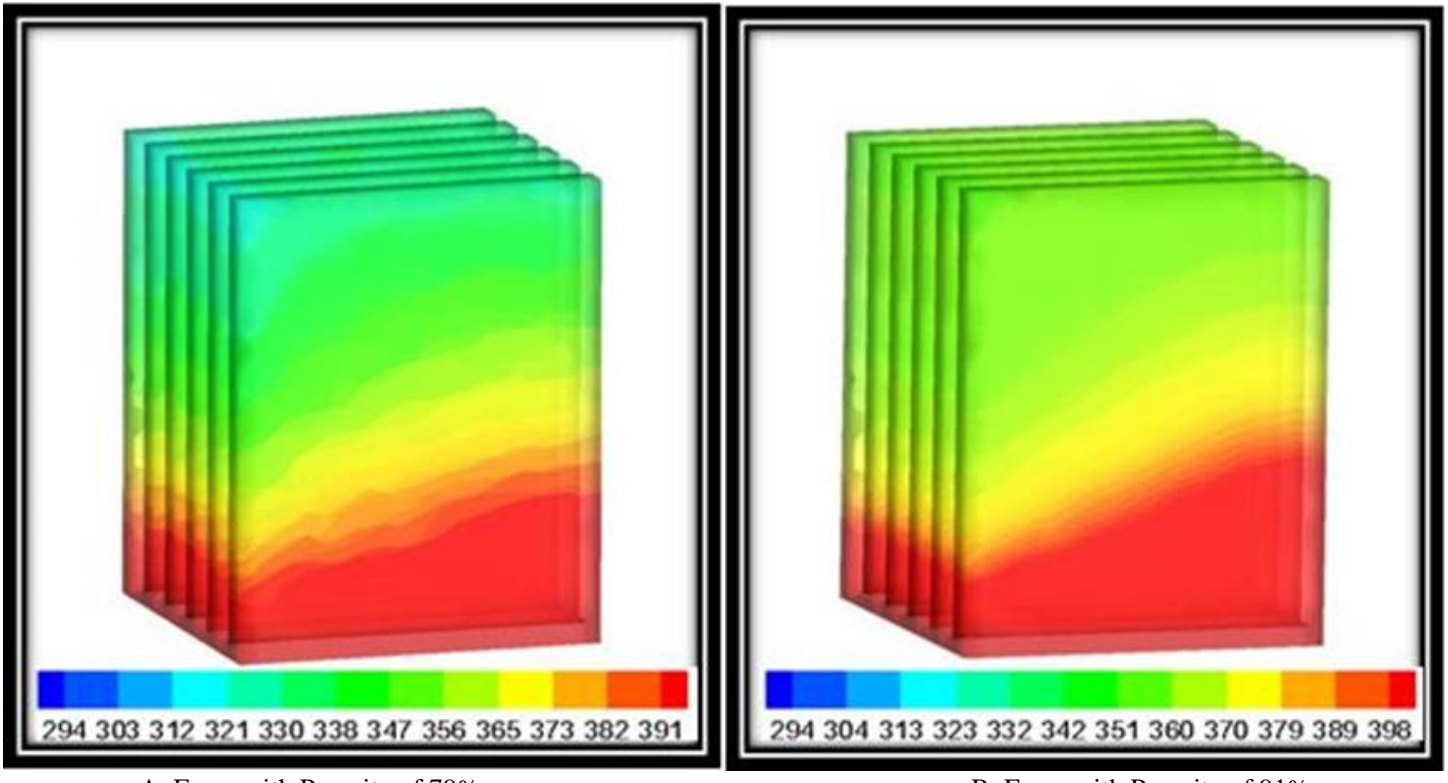

A. Foam with Porosity of $79 \%$

B. Foam with Porosity of $91 \%$

Fig. 11. Contour Temperature (K) of The Heat Sink with Two Types of Porosities, at 30W, 10PPI

The height of the Fins is an important factor in the process of enhancing the thermal performance of the heat sink generally as well as, its contribution in an increasing the fins efficiency to heat dissipation specially. As expected, the height plays a significant role in this purpose because of, the increasing of the fin's height leads to decreasing in the base temperature and thereby the heat dissipation is to be large. Figure 12 shows the impact of the height on base temperature for range of various heights. It is noticed through the figures below that when the ratio $(\mathrm{H} / \mathrm{L})$ is 0.2 the heat dissipation is very bad and this shows clearly in the contour, therefore that at this ratio the base temperature reaches to its highest range. With respect to the case which the ratio equals to 0.6 , the base temperature begins to drop gradually and this meaning the heat dissipation is somewhat better than the dissipation in previous case. The contours in figures(c) and (d), it is explain that by increase this ratio the thermal performance of the metal foam heat sink is enhancing nicely and so as, the dissipation is increase also, when the ratio is changed from 1.0 to 1.4 the base temperature reducing from 391 to 377 $\mathrm{k}$. In the latest two figures (e)and (f), the thermal performance of the heat sink continues to improve as long as there is an increase in this ratio, where the improvement is maximized occurs at 2.2. 


\section{VNJIS Wasit Journal}

VOLUME: ( 7 ), NO.: ( 3 ) 르미므
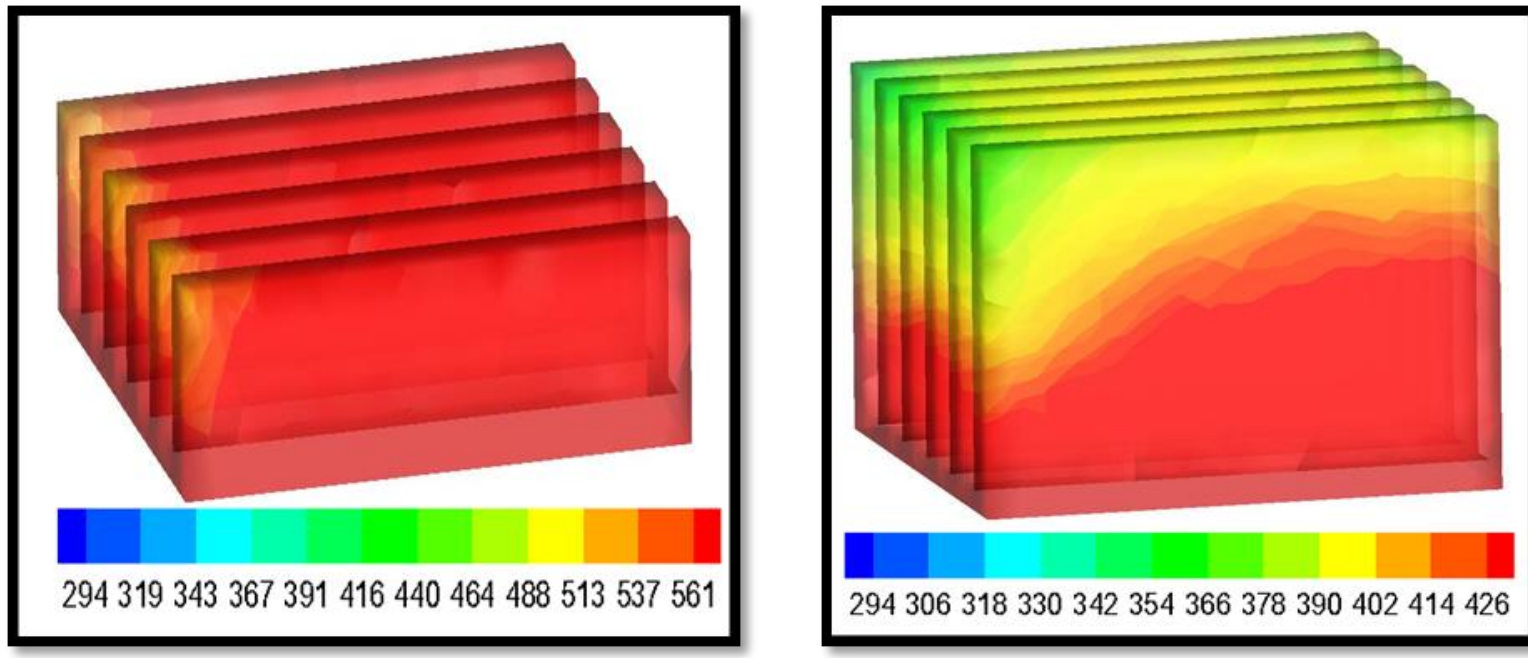

294306318330342354366378390402414426

A. Fins with $(\mathrm{H} / \mathrm{L})=0.2$

B. Fins with $(\mathrm{H} / \mathrm{L})=0.6$
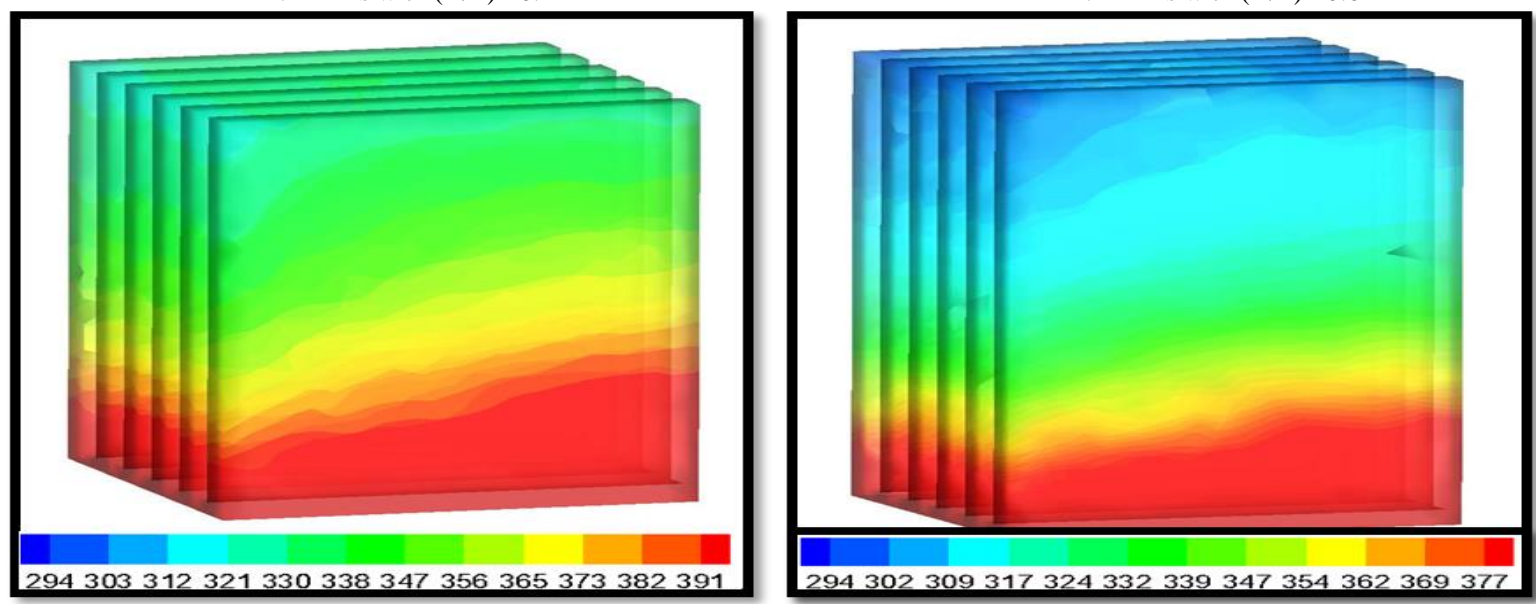

C. Fins with $(\mathrm{H} / \mathrm{L})=1.0$

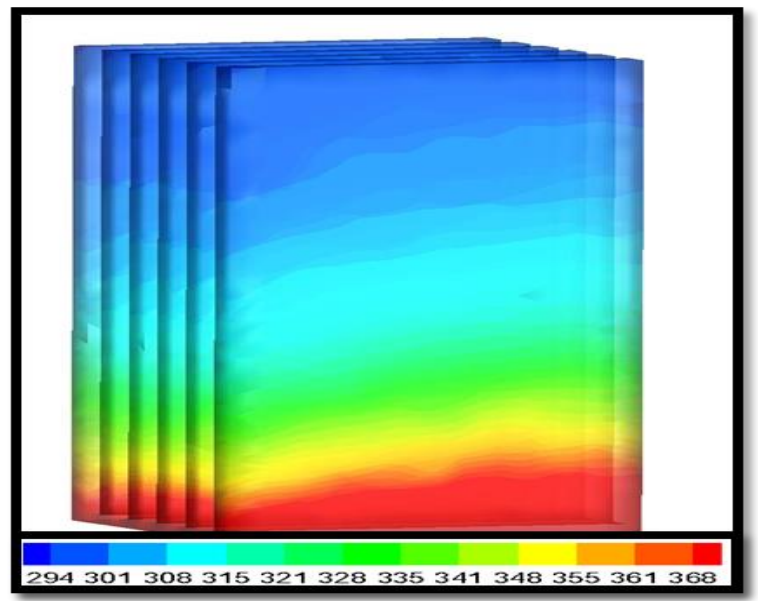

E. Fins with $(\mathrm{H} / \mathrm{L})=1.8$

D. Fins with $(\mathrm{H} / \mathrm{L})=1.4$

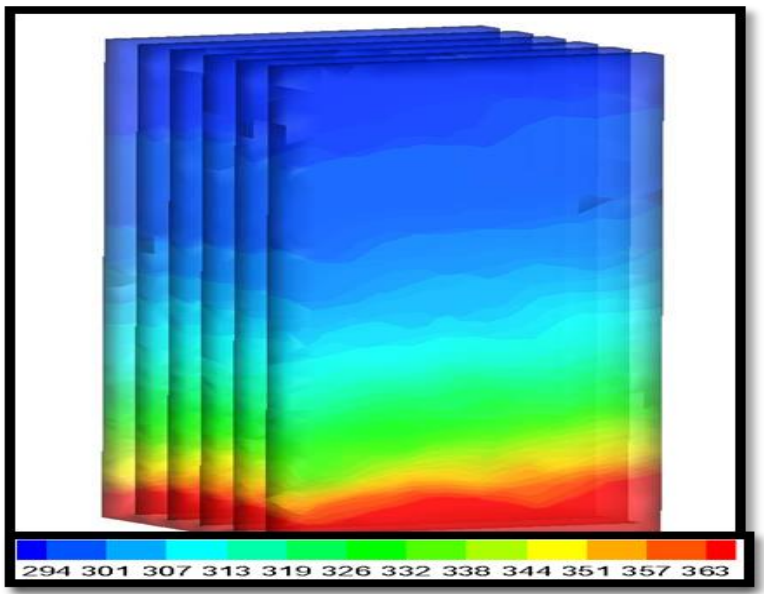

F. Fins with $(\mathrm{H} / \mathrm{L})=2.2$

Fig. 12. Temperature Contours (K) of the Heat Sink with Various Fin Height (a), (b), (c), (d), (e), and (f) of 10PPI, Porosity $79 \%$ and $30 \mathrm{~W}$ 


\section{CONCLUSION}

The thermal performance for heat sink with aluminum metal foam has been investigated under natural convection heat transfer conditions. The study was conducted by using ANSYS Fluent software (R16.1). Various samples with fixed pores per inch of 10 PPI and different of porosities ranging from $95 \%$ to $71 \%$ were investigated based on a model that's depended on a local thermal non-equilibrium (LTNE) in energy equation. Porosity is an important factor effecting on base temperature and enhances the thermal performance of heat sink. Where the enhancement of reduction in base temperature about $(16.7 \%)$, when the porosity reduced from $95 \%$ to $79 \%$, and the maximum enhancement in heat transfer coefficient of porosity $79 \%$ reaches to $21.3 \%$ greater than porosity $95 \%$. Another factor that has proved to be dominant in improving the efficiency of the metal foam heat sink is the ratio of fin height relative to its length, the highest enhancement in base temperature and heat transfer coefficient occurs when the height to length ratio is (2.2) and estimated by $30 \%$ and $83.8 \%$ respectively, compared with the ratio of $(0.2)$.

\section{REFERENCE}

[1] Shih Wei-Hung, Liu Chin-Chia, Hsieh Wen-Hsin, Heat-transfer characteristics of aluminum-foam heat sinks with a solid aluminum core, Int. J. Heat. Mass Transf.

97 (2016) 742-750.

[2] W.H. Hsieh, J.Y. Wu, W.H. Shih, W.C. Chiu, Experimental investigation of heat-transfer characteristics of aluminum-foam heat sinks, Int. J. Heat. Mass Transf. 47

(2004) 5149-5157.

[3] Yunus A. Cengel, Afshin J. Ghajar, Heat and Mass Transfer: fundamentals and Applications, McGraw- Hill Global Education Holdings, LLC, 2016.

[4] X.H. Han, Q. Wang, Y.G. Park, C. T'Joen, A. Sommers, A. Jacobi, A review of metal foam and metal matrix composites for heat exchangers and heat sinks, J. Heat. Transf. Eng. 33 (2012) 991-1009.

[5] A. Bhattacharya, R.L. Mahajan, Finned metal foam heat sinks for electronics cooling in forced convection, J. Electron. Packag. 124 (2002) 155-163.

[6] H. Huisseune, S.D. Schampheleire, B. Ameel, M.D. Paepe, Comparison of metal foam heat exchangers to a finned heat exchanger for low Reynolds number applications, Int. J. Heat. Mass Transf. 89 (2015) 1-9.

[7] S. Mahjoob, K. Vafai, A synthesis of fluid and thermal transport models for metal foam heat exchangers, Int. J. Heat. Mass Transf. 51 (2008) 3701-3711.

[8] S. Feng Feichen Li, Fenghui Zhang, Tian Jian Lu. —-Natural Convection in Metal Foam Heat Sinks with Open Slots, $\| 2017$.

[9] G. Hetsroni, M. Gurevich, and R. Rozenblit, -Natural convection in metal foam strips with internal heat generation, || vol. 32, pp. 1740-1747, 2008.

[10] M. S. Phanikumar and R. L. Mahajan, "Non-Darcy natural convection in high porosity metal foams," vol. 45, pp. 3781-3793, 2002.

[11] P. M. Kamath, C. Balaji, and S. P. Venkateshan, "International Journal of Thermal Sciences Convection heat transfer from aluminium and copper foams in a vertical channel e An experimental study," Int. J. Therm. Sci., vol. 64, pp. 1-10, 2013.

[12] Z. Qu, T. Wang, W. Tao, and T. Lu, "International Journal of Heat and Fluid Flow Experimental study of air 
natural convection on metallic foam-sintered plate," Int. J. Heat Fluid Flow, vol. 38, pp. 126-132, 2012.

[13] M. Paknezhad, A. M. Rashidi, T. Youse, and Z. Saghir, -Case Studies in Thermal Engineering Effect of aluminum-foam heat sink on inclined hot surface temperature in the case of free convection heat transfer, $\|$ vol. 10, no. June, pp. 199-206, 2017. 